\title{
EL SUR DE POZUELOS (PUNA DE JUJUY, ARGENTINA) ANTES DEL 1.000 D.C. PRIMERAS EVIDENCIAS ARQUEOLÓGICAS
}

\section{LTHE SOUTH OF POZUELOS (JUJUY, ARGENTINA) BEFORE 1000 A.D. FIRST ARCHAEOLOGICAL EVIDENCES.}

\section{Carlos Angiorama ${ }^{1}$, Diego Argañaráz Fochi ${ }^{2}$, M. Florencia Becerra ${ }^{3}$, Eze- quiel Del Bel ${ }^{4}$, Osvaldo Díaz ${ }^{5}$, Marco Giusta ${ }^{6}$, Diego Leiton ${ }^{7}$, M. Josefina Pérez Pieroni ${ }^{8}$, Silvina Rodríguez Curletto ${ }^{9}$, Lucrecia Torres Vega ${ }^{10}$}

\author{
1 CONICET-IAM-ISES. San Martín 1545. San Miguel de Tucumán (4000), Tucumán. \\ E-mail: carlosangiorama@gmail.com \\ 2 Facultad de Ciencias Naturales e IML. UNT. Miguel Lillo 205. San Miguel de Tucumán (4000), Tucumán \\ 3 CONICET-IAM-ISES. San Martín 1545. San Miguel de Tucumán (4000), Tucumán. \\ 4 IAM. UNT. San Martín 1545. San Miguel de Tucumán (4000), Tucumán. \\ 5 CONICET-IAM-ISES. San Martín 1545. San Miguel de Tucumán (4000), Tucumán. \\ 6 Facultad de Ciencias Naturales e IML. UNT. Miguel Lillo 205. San Miguel de Tucumán (4000), Tucumán \\ 7 CONICET-IAM-ISES. San Martín 1545. San Miguel de Tucumán (4000), Tucumán. \\ 8 CONICET-IAM-ISES. San Martín 1545. San Miguel de Tucumán (4000), Tucumán. \\ 9 CONICET-IAM-ISES. San Martín 1545. San Miguel de Tucumán (4000), Tucumán. \\ 10. Facultad de Ciencias Naturales e IML. UNT. Miguel Lillo 205. San Miguel de Tucumán (4000), Tucumán
}

Presentado el: 01/06/2013 - Aceptado el: 28/06/2013

\begin{abstract}
Resumen
Este artículo tiene como objetivo dar a conocer las evidencias arqueológicas anteriores al 1.000 d.C. registradas en el sector sur de Pozuelos, las primeras de tal antigüedad reportadas para el área. Estas son escasas en relación a las de épocas posteriores, y consisten en representaciones rupestres, fragmentos cerámicos y material lítico hallado en superficie. No se han detectado hasta el momento ocupaciones en cuevas o aleros, ni arquitectura que puedan asignarse a este período temporal. En base a la información presentada se plantea que la ocupación arcaica de la región habría sido más intensa y extendida que la de época formativa temprana, y que la paulatina disminución en la cantidad de evidencias arqueológicas a medida que avanzamos en el tiempo, se habría acentuado notablemente para el primer milenio de nuestra era. Esto reflejaría un relativo despoblamiento del sur de Pozuelos hasta que, luego del 1.200 d.C., el número de habitantes del área de estudio se habría incrementado exponencialmente.
\end{abstract}

Palabras claves: Pozuelos, Puna de Jujuy, período Arcaico, período Formativo. 


\begin{abstract}
This article aims to present the archaeological evidences prior to $1.000 \mathrm{AD}$ recorded in the area of the South of Pozuelos, the oldest reported until this moment for the region. These are less abundant compared to those of recent times, and include rock art, pottery fragments and lithic materials found on the surface. We have not detected so far neither human occupations in caves or rockshelters, nor architecture that can be assigned to this temporary period. Based on the information presented it is suggested that the archaic occupation of the region would have been more intense and widespread than the early formative presence, and that the gradual decrease in the amount of archaeological evidence as we move forward in time, would have considerably accelerated in the first millennium $A D$. This would reflect a relative depopulation of the South of Pozuelos until 1.200 AD, when the number of inhabitants in the area of study would have exponentially increased.
\end{abstract}

Keywords: Pozuelos, Puna of Jujuy, Archaic period, Formative period.

\title{
Introducción
}

El bolsón de Pozuelos (Jujuy, Argentina) forma parte del extremo meridional del altiplano andino. Se trata de una cuenca endorreica delimitada, en su porción sur, por las sierras de Rinconada y Carahuasi (al oeste), Cochinoca (al este) y Quichagua (al sur). Hacia el norte, la depresión ingresa en territorio boliviano sin solución de continuidad. El relieve es en parte plano o con bajas ondulaciones, y en parte abrupto y quebrado. Los escasos cursos de agua permanentes y sus afluentes temporarios desembocan en la Laguna Pozuelos, base del bolsón, cuya cota se ubica en los $3660 \mathrm{msnm}$. Los cerros más altos, los de la serranía de Carahuasi, alcanzan una altura de $5100 \mathrm{msnm}$. El área presenta un clima continental, con una gran amplitud térmica diaria, fuertes vientos y precipitaciones estivales que oscilan entre los 400 y $200 \mathrm{~mm}$ anuales, con una disminución general en sentido noreste-sudoeste, aunque fuertemente modificada por elementos orográficos. Sin embargo, las precipitaciones son muy irregulares a lo largo de los años (Coira 1979; Tecchi 1991).

En 2005 iniciamos nuestras investigaciones en el sector sur de Pozuelos con dos objetivos últimos: evaluar el rol desempeñado por las comunidades agropastoras del área en el tráfico prehispánico interregional, y contrastar nuestras propuestas, generadas desde la Quebrada de Humahuaca (Jujuy), acerca de las características que habrían asumido la explotación y la circulación de minerales metalíferos en el extremo noroccidental de Argentina durante época prehispánica tardía y colonial temprana (Angiorama 2001, 2003, 2006). De esta porción del altiplano de unos 40 × 40 km (ver Figura 1), tan sólo el Pucará de Rinconada, localizado en los márgenes del área, había sido objeto de investigaciones arqueológicas con anterioridad (Ambrosetti 1902; Alfaro y Suetta 1970; Boman 1992 [1908]; Ruiz 1996; Ruiz y Albeck 1997; Suetta y Alfaro 1979). Sin embargo, a pesar de que casi nada se sabía acerca de su historia prehispánica, había ciertos elementos que la convertían en un área particularmente interesante para evaluar diversos aspectos vinculados con procesos de interacción regional y de producción minero-metalúrgica (ver Angiorama 2009).

Las tareas realizadas hasta el momento estuvieron orientadas a formular un marco cronológico preliminar para la etapa agropastoril en la zona, identificar tendencias en los modos de utilización del espacio regional en cada momento, establecer relaciones entre las ocupaciones del sur de Pozuelos y regiones vecinas, y formular modelos explicativos de los modos de uso del territorio identificados y sus transformaciones. Comenzamos los trabajos de campo 
realizando prospecciones arqueológicas extensivas e intensivas, efectuando relevamientos y muestreos superficiales de cada uno de los sitios hallados, registrando la distribución de los recursos naturales disponibles en los sectores prospectados, y muestreando las fuentes de minerales metalíferos y materias primas líticas presentes (Angiorama 2009). Los trabajos de campo tuvieron lugar en tres sectores diferentes:

-en diversas porciones del "fondo de la cuenca", a unos 3.700-3.800 msnm, donde prospectamos parte de las márgenes del Río Cincel y cauces subsidiarios, y casi todas las terrazas de ignimbritas y cerros aislados localizados en el centro del área (como los cerros Pan de Azúcar, León Grande, León Chico, etc.);

-en una "franja de altura", localizada hacia el oeste del área de estudio, a unos 4.000-4.200 msnm, donde se ubican una serie de lagunas, ciénegas y peñas distribuidas a lo largo de una gran falla paralela a la Sierra de Carahuasi, desde su extremo sur hasta laguna Pampa Colorada, al norte de la localidad de Santo Domingo; y

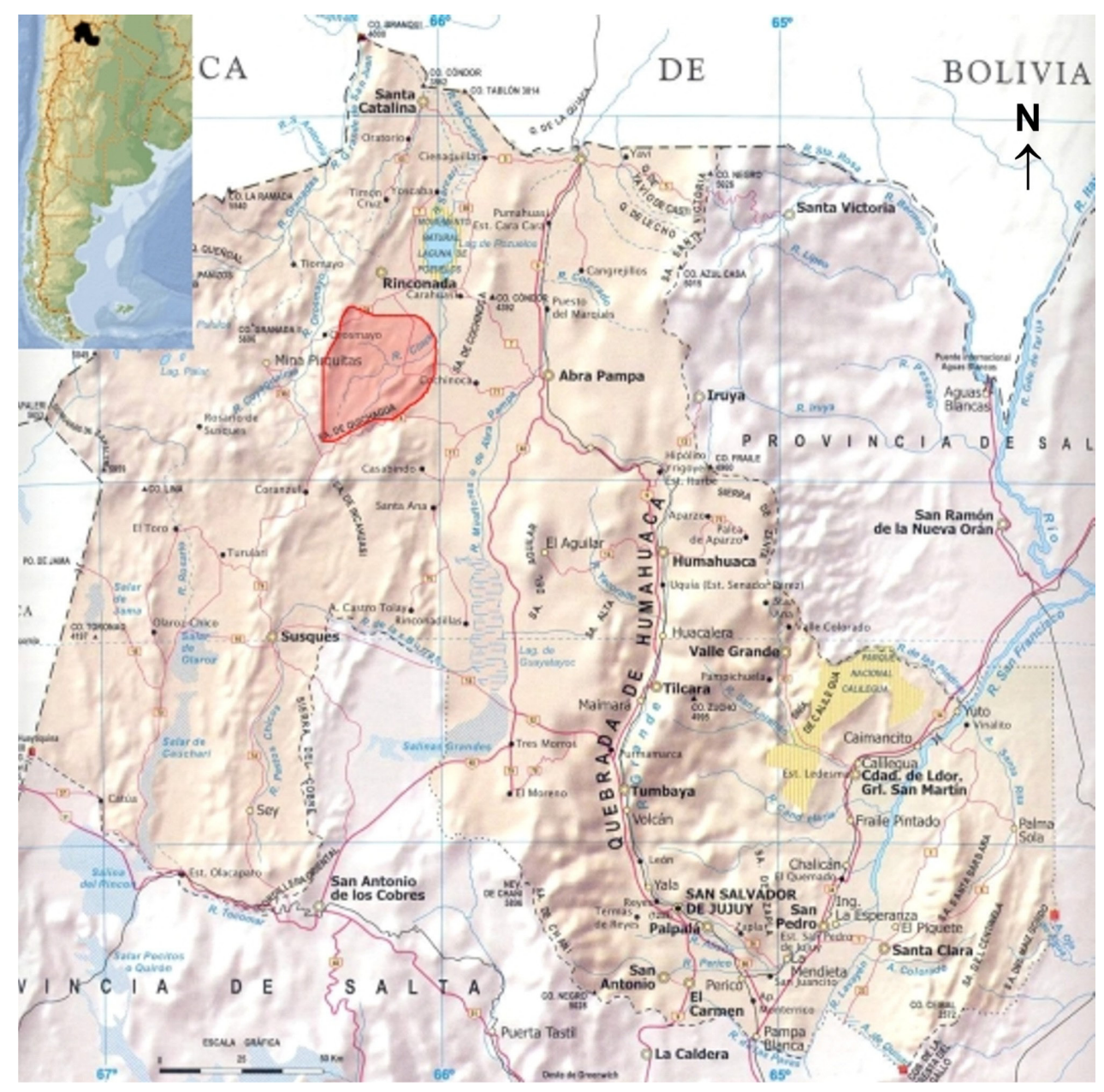

Figura 1. Mapa de ubicación del área de estudio (sector sombreado). La escala gráfica representa $50 \mathrm{~km}$. 
-en casi todas las "quebradas" que comunican el fondo de la cuenca con la franja de altura mencionada y, más al oeste, con la cuenca del Río Orosmayo y Vilama, y todas las quebradas que comunican Pozuelos con Rachaite y Doncellas, hacia el sur.

Las tareas realizadas nos permitieron esbozar un primer panorama de la arqueología del área, habiéndose registrado más de trescientos sitios arqueológicos. Luego, de acuerdo con los objetivos del Proyecto, fueron seleccionadas para ser excavadas y sondeadas una serie de estructuras localizadas en diversos sectores del área. Hasta el momento hemos excavado catorce recintos completos, y sondeamos ocho recintos más, un alero, dos cuevas y tres refugios construidos bajo aleros o al pie de grandes paredones, localizados en su mayoría entre estructuras de cultivo (Angiorama 2009).

Si bien el Proyecto está orientado al estudio de las sociedades prehispánicas agroalfareras que habitaron el sur de Pozuelos y la posterior ocupación colonial del área, durante las intensas prospecciones arqueológicas realizadas hemos localizado y registrado una serie de hallazgos más antiguos. El objetivo de este artículo es dar a conocer las evidencias arqueológicas que datarían de antes del 1.000 d.C., límite temporal de las Jornadas1 en la que este trabajo fue expuesto originalmente. Esta presentación tiene algún valor, a nuestro criterio, por el hecho de que se trataría de las primeras evidencias de tal antigüedad reportadas para el sur de Pozuelos.

Somos conscientes, sin embargo, de que para poder luego evaluar la significación de los hallazgos arqueológicos superficiales efectuados en diversos sectores de nuestra área de estudio, es necesario conocer las fluctuaciones climáticas ocurridas a lo largo del tiempo y los procesos geomorfológicos y tafonómicos generales que han actuado en ella. Cuando se intentan abordar aspectos que requieren una visión espacial amplia y que han ocurrido a lo largo de un lapso temporal muy largo, esto resulta aún más imprescindible. Sin embargo, hasta el momento no existen estudios publicados que traten la incidencia de estos procesos sobre el registro arqueológico para esta zona específica de la Puna jujeña, y no se conocen tampoco las características paleoambientales de la misma. Sabemos que a nivel regional el ambiente osciló entre condiciones húmedas y áridas a lo largo del Holoceno. Sin embargo, estas condiciones no se dieron de manera homogénea para toda el área, tanto en su duración como en la intensidad de los procesos. En términos generales, entre el $9.000 \mathrm{AP}$ y el 6.500 AP se registraron condiciones húmedas que culminaron con un pico de aridez entre el 6.500 AP y el 5.200 AP. El ambiente se habría recuperado luego hacia el $2.700 \mathrm{AP}$, hasta el 1.600 AP. En períodos posteriores se produjo un cambio abrupto, marcado por un descenso de la humedad, con una fase de mayor sequedad registrada para aproximadamente el 1.000 AP. Esto coincidiría con la anomalía climática medieval, registrándose hasta el $600 \mathrm{AP}$ y volviendo luego a condiciones más húmedas hasta el 500 AP (Lupo et al. 2007; Olivera et al. 2004). En la actualidad, el área de estudio se localiza en un ambiente árido, con una gran amplitud térmica diaria, y caracterizado por bajas precipitaciones y vientos fuertes. Como resultado, la cobertura vegetal que presenta es escasa (Coira 1979).

Estas oscilaciones climáticas seguramente afectaron de diversas maneras la visibilidad de las evidencias arqueológicas y los procesos de formación del registro artefactual. En los períodos de retroceso del nivel lacustre se habrían incrementado los procesos erosivos eólicos. En momentos más húmedos habrían predominado los de depositación o agregación de sedimentos en los fondos de cuencas, mientras que en quebradas y laderas la erosión hídrica debió haber repercutido en mayor grado que en momentos áridos. 
Dadas estas características ambientales pasadas y presentes, los factores posdepositacionales que pudieron haber actuado sobre los materiales arqueológicos depositados debieron incluir predominantemente procesos de meteorización mecánica, como la crioclastía, erosión eólica, la formación de cárcavas y barrancos, entre otros. A ellos se suman, obviamente, las actividades antrópicas llevadas a cabo por los habitantes pasados y actuales del área, cuyo número parece haberse incrementado exponencialmente entre el 1.200 d.C. y la conquista española, fundamentalmente en el fondo de la cuenca (Angiorama 2010). Las actividades productivas desarrolladas, sobre todo las agrícolas y ganaderas (ampliamente extendidas en época prehispánica tardía), seguramente han sido factores sumamente relevantes en la conformación del registro arqueológico que hoy encontramos, especialmente en las terrazas y cerros del fondo de la cuenca. Las quebradas y la franja de lagunas y ciénegas de altura, en cambio, habrían recibido un impacto antrópico menor a lo largo de la historia.

Teniendo presente las limitaciones que provoca esta falta de estudios geomorfológicos, paleoambientales y tafonómicos locales, presentamos a continuación los hallazgos arqueológicos más antiguos efectuados en el sur de Pozuelos.

\section{Los hallazgos más antiguos}

En realidad, las evidencias arqueológicas anteriores al 1.000 d.C. que hemos hallado mediante los trabajos de campo realizados son escasas en relación a las de épocas posteriores. En primer lugar, no hemos encontrado ocupaciones en cuevas o aleros que daten del período en cuestión. La única posible excepción quizás la constituya una pequeña cueva con arte arcaico en su interior y un alero aledaño, ambos sondeados recientemente y con fechados radiocarbónicos en proceso (Río Herrana 14, ver más adelante). Tampoco hemos registrado arquitectura que podamos asignar al primer milenio de la era o a momentos anteriores. Ninguno de los recintos que hemos excavado o sondeado es anterior al 1.200 d.C. Lo mismo ocurre con la ocupación de los refugios sondeados. Las evidencias asignables al periodo abordado en este artículo consisten únicamente en representaciones rupestres, fragmentos cerámicos y material lítico hallados en superficie.

\section{Las representaciones rupestres}

Los trabajos de campo realizados hasta el momento nos han permitido detectar y registrar 25 sitios arqueológicos con representaciones rupestres no publicados en la literatura arqueológica regional. Éstas presentan una alta variabilidad tanto en los patrones de diseño intra e intersitio, como en el emplazamiento de los mismos. Sin embargo, a pesar de esta variabilidad, existe un predominio de dos motivos principales: el camélido y la figura humana. A pesar de que estos dos motivos se presentan en casi todos los sitios, existe una clara distinción entre ellos en cuanto a la frecuencia con la que aparecen dentro del mismo: el camélido, ya sea aislado, en grupo o en caravana, se presenta con una alta frecuencia en cada sitio, mientras que la figura humana aparece con una frecuencia claramente más baja. En menor proporción se encuentran otros diseños y motivos zoomorfos, puntiformes, geométricos, uncus y escutiformes, observándose en muchos casos una reutilización de los paneles, evidenciada por las superposiciones de motivos y por las variaciones tonales en las pátinas de los grabados y en las mezclas pigmentarias. Siete de los 25 sitios mencionados, presentan motivos que estarían ubicados cronológicamente en el rango temporal tratado en este trabajo. Ellos son los descriptos a continuación. 


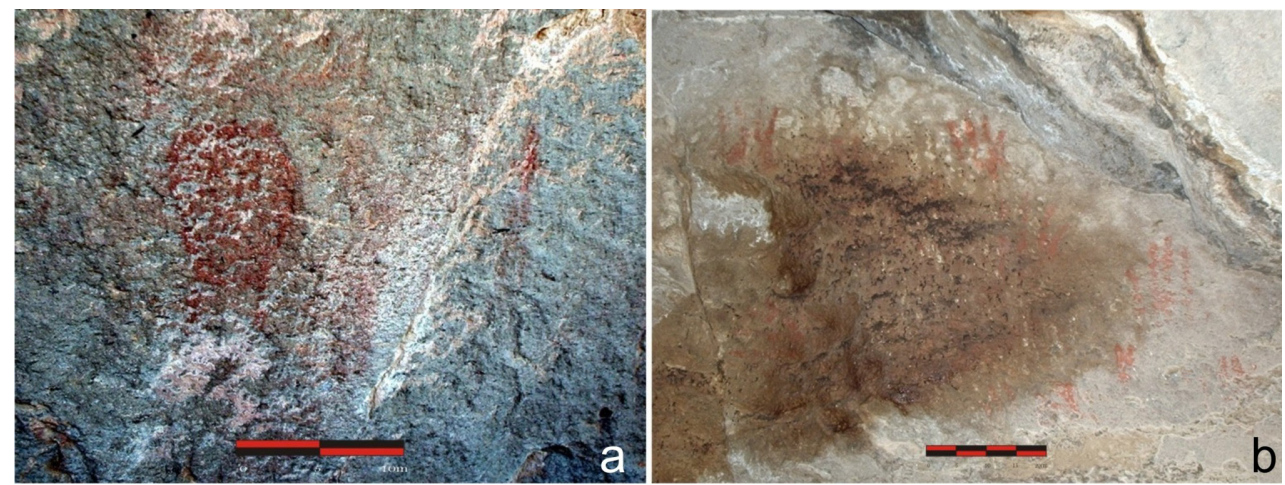

Figura 2. Representaciones rupestres. Referencias: a: Ciénega Rodeo 1-15 (CIR 1-15). La escala gráfica representa $10 \mathrm{~cm}$; b: Río Herrana 14 (RH 14). La escala gráfica representa $20 \mathrm{~cm}$.

El sitio Río Herrana 14 consiste en un panel ubicado en el interior de una cueva emplazada en la cima de una terraza, en el fondo de la cuenca. En el panel se observan trazos lineales pintados en rojo, de los cuales al menos algunos de ellos estarían representando manos esquemáticas en positivo (ver Figura 2.b.). En un sondeo realizado recientemente en el interior de la cueva detectamos dos niveles de ocupación separados por un largo período de abandono. A juzgar por unas gotas de pigmento similar al utilizado para confeccionar los motivos descriptos, halladas en el nivel más antiguo, éste correspondería a la ocupación durante la cual se ejecutaron las pinturas. A las gotas de pigmento se asociaban únicamente tres fragmentos óseos de Artiodactyla (Norma Nasif comunicación personal 2009), uno de los cuales se encuentra actualmente siendo procesado para la obtención de un fechado radiocarbónico. Provisoriamente, estimamos que las pinturas datarían de época arcaica temprana.

El sitio Peña Colorada 1 presenta un panel vertical ubicado en un afloramiento rocoso localizado en la cabecera de una de las quebradas que conectan Pozuelos con el sur, junto al abra. En el panel se observa un motivo antropomorfo pintado en rojo desvaído con cuello largo y cabeza no observable debido al estado de conservación, dos brazos muy largos extendidos hacia los costados y dos piernas rectas hacia abajo, con representación de posible falo entre ellas (ver Figura 3.b.). Esta figura está acompañada de 41 puntos pintados en rojo que se encuentran sobre el cuerpo y por debajo de los brazos del la misma, y presentan un tono más oscuro que el que se observa en el antropomorfo. Podemos vincular el motivo pintado con otros similares que datan de época arcaica tardía (Aschero y Podestá 1986). En el lugar se halló material lítico de época arcaica en superficie, y ocho depósitos sobre nivel que datarían de época colonial (Angiorama 2010).

El sitio Almacén Cincel 18 presenta un panel vertical emplazado en una de las caras de un gran bloque rocoso localizado en la cima de una lomada, en el fondo de la cuenca. El panel presenta seis motivos pintados (ver Figura 3.d.). El primero se trata de un motivo zoomorfo pintado en rojo con vista hacia la izquierda, cortado por una grieta en su parte posterior. Se identifican en él tres patas, cuello, cabeza y oreja curva hacia atrás, elementos que nos permiten considerarlo tentativamente de época arcaica tardía (Carlos Aschero, comunicación personal 2008). El segundo se trata de un motivo de grandes dimensiones que presenta una combinación de rojo y amarillo, del cual muchas partes no pueden identificarse por el grado de erosión que presenta. El tercero, se trata de un posible antropomorfo de color rojo, del que pueden identificarse, debido al grado de erosión, sólo parte del torso, el brazo izquierdo y las dos piernas rectas hacia abajo. El cuarto y quinto motivo son líneas 


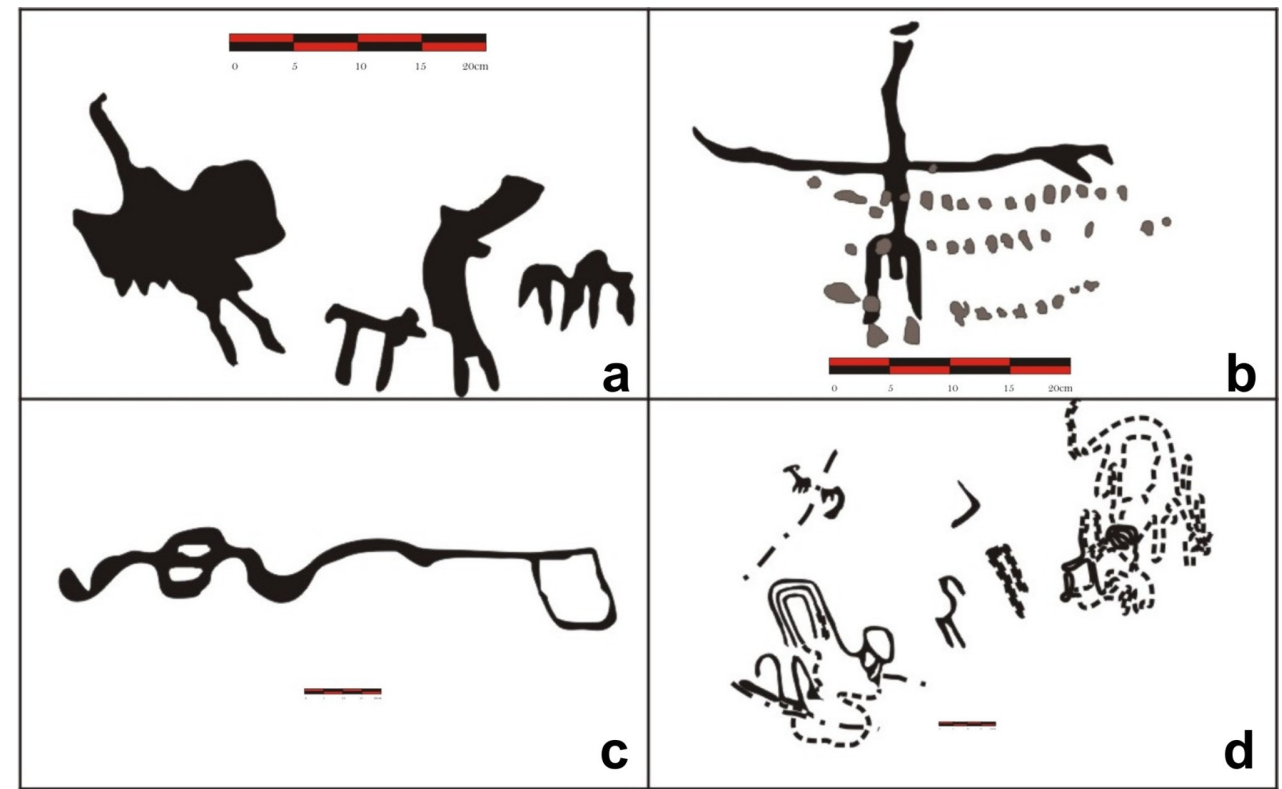

Figura 3. Representaciones rupestres. Referencias: a: Río Herrana 5 (RH 5); b: Peña Colorada 1 (PC 1); c: Casa Colorada 1-2 (CCO 1-2); d: Almacén Cincel 18 (AC 18). Las escalas gráficas representan 20 cm en todos los casos.

aisladas de color rojo, y el sexto motivo, también de color rojo y de grandes dimensiones es indeterminado. No se ha hallado material arqueológico en superficie, pero sí otros bloques cercanos similares con motivos tardíos pintados (posteriores al 1.000 d.C.).

El sitio Río Herrana 5 presenta un panel ubicado en una oquedad formada en un bloque rocoso localizado en la parte alta de una terraza de ignimbrita, en el fondo de la cuenca. El panel está compuesto por cuatro motivos pintados en rojo desvaído (ver Figura 3.a.). El primero, de mayor tamaño, consiste en un motivo posiblemente zooantropomorfo. El segundo se trata de un camélido esquemático con dos patas largas en relación al cuerpo, cola curva hacia abajo y oreja curva hacia adelante, elementos que nos permiten postular su probable adscripción temporal al Arcaico Tardío o inicios del Formativo (Aschero 1979). El tercer motivo es un posible antropomorfo que presenta dos piernas rectas hacia abajo y quizás un brazo. Debido al estado de conservación no se identifica el sector izquierdo-superior de la figura. Finalmente, el cuarto consiste en un motivo peiniforme, con cinco dientes hacia abajo. En el lugar abundan estructuras y material arqueológico de época tardía. Además de este panel, se pudieron observar otros dos de similares características en cuanto a ubicación en oquedades en las cercanías del sitio, pero se encuentran en muy mal estado de conservación, por lo que no pudieron identificarse los motivos.

El sitio Casa Colorada 1-2 se trata de un panel vertical inclinado, ubicado en un afloramiento rocoso localizado en el fondo de la cuenca, cercano a un paso importante que comunica Pozuelos con el sur. En el panel se encuentra un motivo ejecutado mediante la técnica de grabado, compuesto por un rectángulo del cual se proyecta hacia la izquierda un serpentiforme de gran tamaño (ver Figura 3.c.). Ambos elementos presentan una pátina fuerte, y pueden ser asignados tentativamente a época formativa temprana (Carlos Aschero, comunicación personal 2008). En bloques contiguos se ha hallado abundante arte tardío grabado y pintado, y en los alrededores se encuentra arquitectura tardía y material lítico arcaico, formativo temprano, y tardío (posteriores al 1.000 d.C.). 
El sitio Ciénega Rodeo 1-15 presenta un panel vertical ubicado en un afloramiento rocoso localizado junto a una gran ciénega de altura $(4.000 \mathrm{msnm})$. En él se identifican dos motivos antropomorfos pintados en rojo (ver Figura 2.a.). El motivo de la izquierda, presenta torso grande y rectangular relleno y piernas rectas hacia abajo. La cabeza y los brazos no se distinguen claramente, pero puede que presente un posible tocado que cae hacia la derecha del cuerpo. El motivo de la derecha presenta dos piernas rectas hacia abajo y dos brazos cortos extendidos hacia los costados. Por encima de éstos, se distinguen dos manchas difusas rojas, y en el extremo derecho del panel se observa lo que posiblemente puedan ser líneas acompañadas de una probable garra de ave. El motivo antropomorfo de la derecha podría tratarse de uno de los del Grupo estilístico B de Inca Cueva, de época formativa temprana (Aschero 1979). Cerca de este panel se halló arte tardío pintado y materiales arqueológicos que evidencias ocupaciones arcaicas, formativas tempranas, y tardías (posteriores al 1.000 d.C.).

El sitio Río Candado 17 se trata de un panel vertical inclinado, ubicado en una roca plana de gran tamaño que se encuentra apoyada sobre un afloramiento rocoso, en la cima de una terraza localizada en la base de una de las quebradas que comunican Pozuelos con el sur. En el panel se encuentran dos camélidos ejecutados mediante la técnica de picado en surco con un tono de pátina fuerte (ver Figura 4). Uno de ellos tiene cuatro patas y el otro dos. Ambos presentan dos orejas dispuestas a $45^{\circ}$, el torso curvo, convexo hacia arriba y la cola larga y recta, también hacia arriba. Por otro lado, se observó un panel enfrentado a éste, en el cual se pudieron identificar una máscara completa y otra similar pero sin terminar, además de tres pares de huellas de camélidos ubicados en forma paralela y ascendente. Estos motivos se encuentran ejecutados mediante la técnica de picado en surco. De acuerdo a las características de los motivos y el tono de la pátina pueden ser considerados provisoriamente de época formativa en el contexto local, pero hay algunos

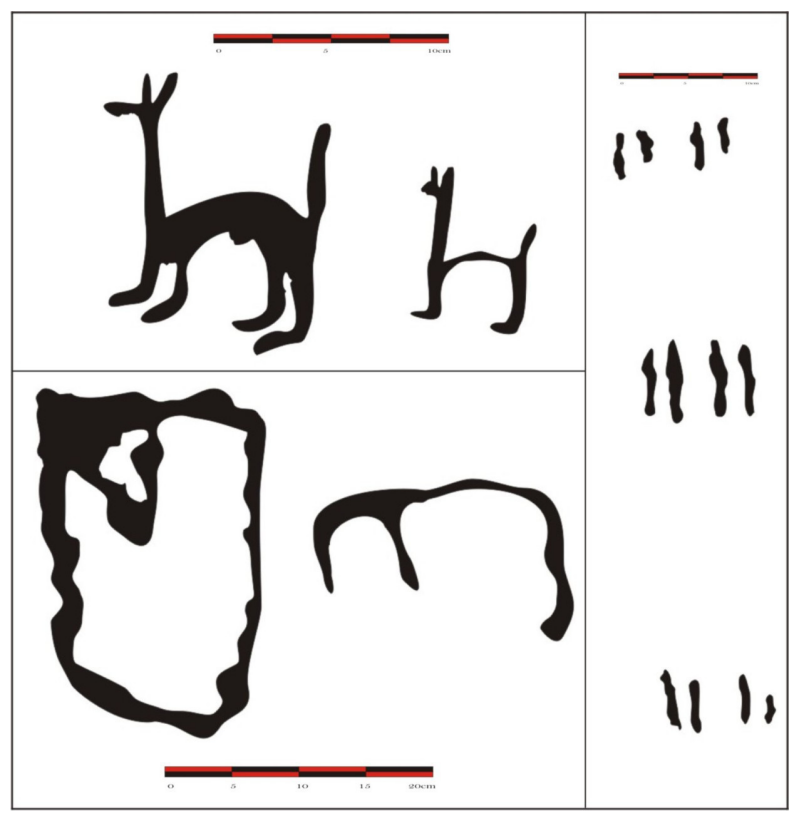

Figura 4. Representaciones rupestres. Río Candado 17. Las escalas gráficas superiores representan $10 \mathrm{~cm}$, mientras que la inferior representa $20 \mathrm{~cm}$. 
motivos similares en el norte del río Loa (Chile) que datan de época más tardía (Carlos Aschero, comunicación personal 2008; Aschero 2007). La roca grabada se encuentra junto a un alero, pero no se ha hallado en el lugar material arqueológico en superficie.

En síntesis, hemos hallado tres sitios con representaciones asignables al Arcaico, una al Arcaico Tardío o al Formativo Temprano, y tres al Formativo. En todos los casos los paneles presentan muy pocos motivos representados. Los camélidos y los antropomorfos se encuentran en proporciones similares, a diferencia de lo que ocurre en el área con el arte posterior al 1.000 d.C., donde los primeros son mucho más abundantes que las figuras humanas, o que cualquier otro motivo. En el arte arcaico y formativo del sur de Pozuelos los camélidos no forman grupos ni caravanas como en momentos tardíos. Por el contrario, se encuentran solos o, en un solo caso, dos ejemplares juntos.

Ninguno de los siete sitios mencionados presenta una alta visibilidad de las representaciones, siendo en tres de ellos, en realidad, muy poco visibles los paneles con arte. No hemos hallado reutilización de paneles para la ejecución de motivos en épocas diferentes, como sí ocurre luego del 1.000 d.C. Sólo tres de los siete sitios con arte arcaico y formativo tienen cerca arte tardío, pero nunca ejecutado en los mismos paneles. En los cuatro sitios restantes no se ha vuelto a pintar o grabar en épocas posteriores.

\section{La cerámica}

De los aproximadamente 3.500 fragmentos de cerámica que hemos recuperado mediante las prospecciones y excavaciones realizadas, la enorme mayoría no presenta tratamientos de superficie que resulten diagnósticos temporales. De la porción de fragmentos con decoración, una gran cantidad corresponde a los estilos tardíos llamados Chicha Morado (con o sin inclusiones blancas) y Agua Caliente Pintado o Queta Negro sobre Rojo o Casabindo Negro sobre Rojo (Ottonello 1973; Ottonello y Krapovickas 1973; Raffino et al. 1986). En mucha menor proporción hemos registrado fragmentos de otros estilos del segundo milenio de nuestra era, tales como los denominados Pozuelos con Cuarzo, Agua Caliente Rojo Pulido, Portillo Morado sobre Ante, Peñas Coloradas, y variantes locales de aribaloides incaicos (Krapovickas 1975; Raffino et al. 1986). Por el contrario, no hemos hallado ni un solo fragmento que podamos asignar con seguridad a los estilos cerámicos diagnósticos de épocas anteriores al 1.000 d.C., tales como San Pedro Negro Pulido, San Francisco, El Morro, Cerro Colorado Polícromo, Cerro Colorado Inciso, Cristóbal Imbricado y Alfarcito Bicolor (Agüero et al. 2006; Fernández 1996; Nielsen 1997; Ottonello y Krapovickas 1973; Sinclaire 2004). Tan sólo dos fragmentos cerámicos podrían ser las excepciones:

-uno de paredes finas, de pasta grisácea, superficie externa negro pulida e interior negro alisado que podría tratarse de un fragmento de estilo Séquitor Gris Pulido (Axel Nielsen, comunicación personal 2008), abundante en el Loa Superior y el oasis de Atacama durante el primer milenio de nuestra era (Agüero et al. 2006, Sinclaire 2004) (ver Figura 5.b.); y

-otro con ambas superficies alisadas, con una fina capa de engobe ante en la cara externa, sobre la cual se pintaron líneas finas de color morado oscuro formando una figura angular romboide, dentro de la cual hay pintados varios lunares morados, que podría tratarse de un fragmento de estilo Yura Poligonal (Axel Nielsen, comunicación personal 2008), del Período Medio en los Valles Potosinos y oriente del Altiplano de Uyuni-Oruro (Lecoq 2001) (ver Figura 5.a.). 
Sin embargo, el hecho de tratarse de tan sólo dos fragmentos de una muestra de más de 3.000, y la circunstancia de haber sido ambos hallados en superficie, $y$, en el caso del primero, en un contexto posiblemente tardío (Angiorama 2010), no nos permite efectuar mayores consideraciones sobre la antigüedad o la significación de estos hallazgos.

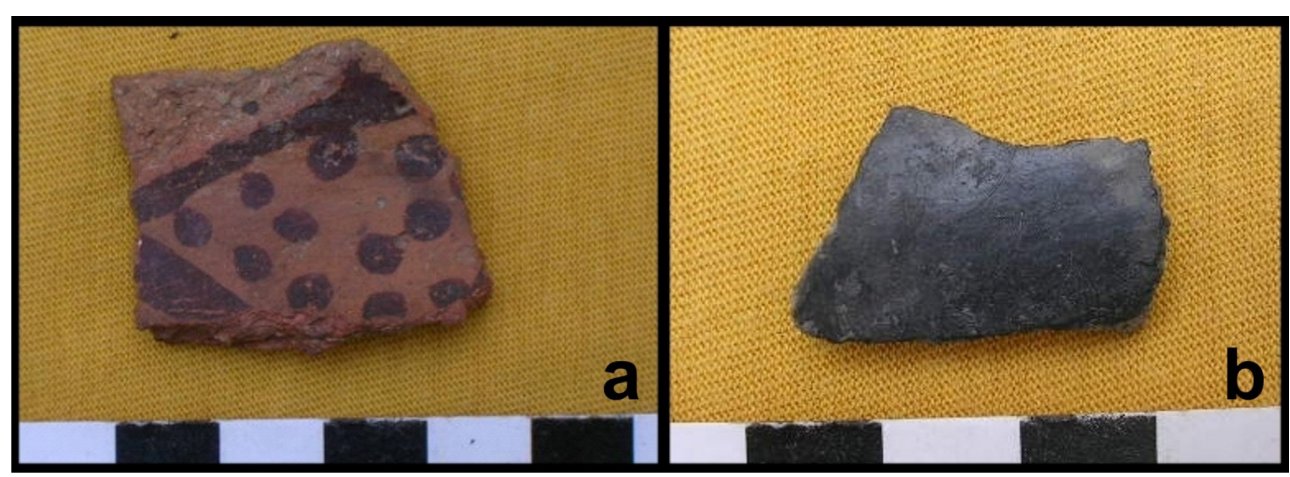

Figura 5. Fragmentos cerámicos. Referencias: a: fragmento de estilo similar al Yura Poligonal; b: fragmento de estilo similar al Séquitor Gris Pulido.

\section{El material lítico}

Por último, las prospecciones realizadas nos permitieron hallar en superficie diversos tipos de puntas de proyectil líticas que, por haber sido fechadas en otros contextos de la Puna argentina y chilena, pueden considerarse indicadores cronológicos de cierta precisión. Cabe destacar que en algunos casos, estas puntas se encontraron asociadas a otros tipos de artefactos líticos que aún no han sido analizados. De un conjunto de unas 230 puntas de proyectil recuperadas mediante las prospecciones llevadas cabo hasta el momento, hemos seleccionado 99 que responden a patrones típicos del rango temporal que nos incumbe. De manera provisoria las hemos clasificado en seis tipos diferentes (ver Figura 6), siguiendo las pautas propuestas para hallazgos similares efectuados en Quebrada de Humahuaca (Jujuy), Sierra de Aguilar (Jujuy) y cuenca de Antofagasta de la Sierra (Catamarca):

1) Triangulares de base recta y sección delgada (24 ejemplares), fechadas aproximadamente entre el 10.500 AP y el 9.000 AP (Arcaico Temprano) (Martínez 2003);

2) Lanceoladas grandes (de más de $5 \mathrm{~cm}$ de largo y más de 1,5 cm de ancho) (33 ejemplares), entre el 8.000 AP y el 7.000 AP (Arcaico Medio) (Hocsman 2006, 2010; Martínez 2003);

3) Lanceoladas con espolones (cinco ejemplares), entre el 8.000 AP y el 7.000 AP (Arcaico Medio) (Jorge Martínez, comunicación personal 2008);

4) Lanceoladas pequeñas (de hasta $5 \mathrm{~cm}$ de largo y hasta $1,5 \mathrm{~cm}$ de ancho) (19 ejemplares), entre el 4.000 AP y el 2.900 AP (Arcaico Tardío) (Hocsman 2006, 2010);

5) Pedunculadas de limbo triangular de lados convexos (13 ejemplares), entre el $3.200 \mathrm{AP}$ y el 2.500 AP (Formativo Temprano) (Hocsman 2006, 2010); y 


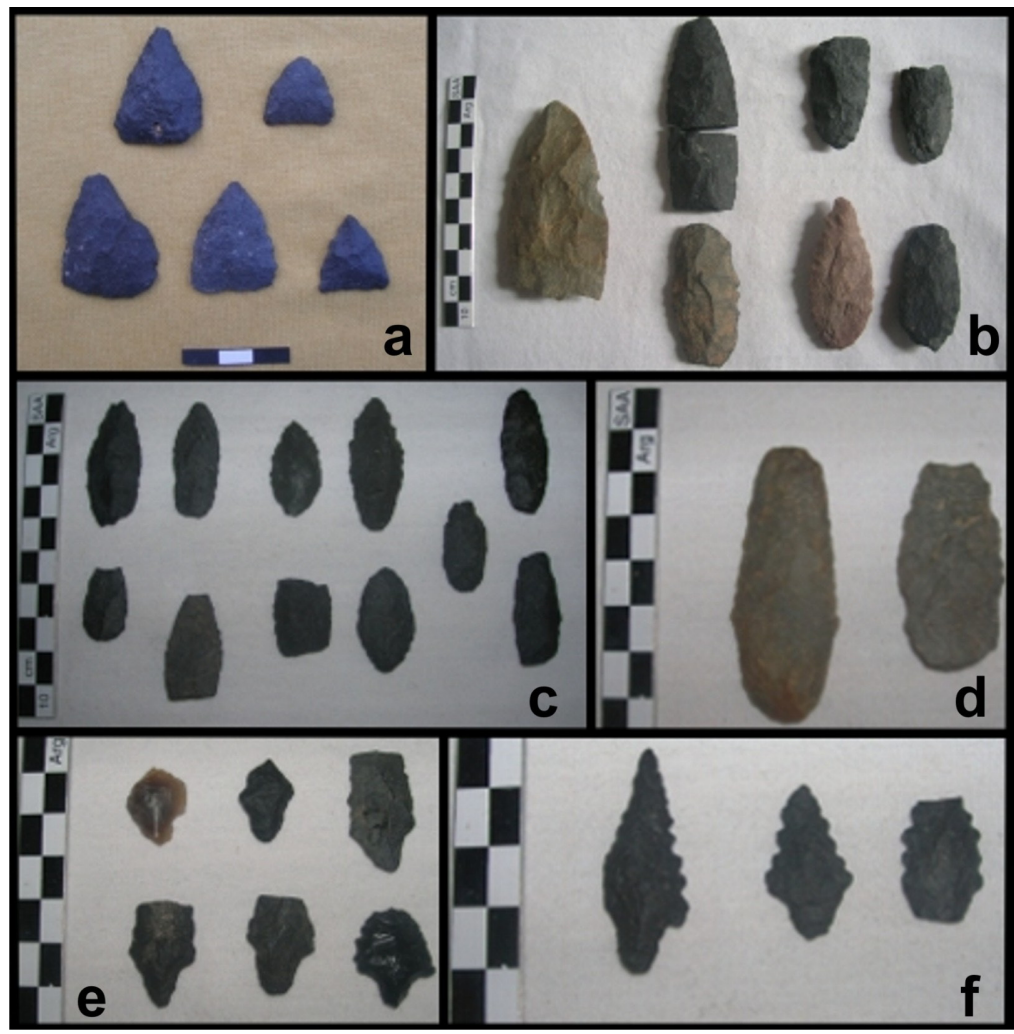

Figura 6. Puntas de proyectil. Referencias: a: triangulares de base recta; b: lanceoladas grandes (largo $>$ a $5 \mathrm{~cm})$; c: lanceoladas pequeñas (largo < a $5 \mathrm{~cm}$ ); d: lanceoladas con espolones; e: pedunculadas de limbo triangular de lados convexos; f: pedunculadas de limbo triangular de lados rectos y bordes dentados.

6) Pedunculadas de limbo triangular de lados rectos y bordes dentados (5 ejemplares), entre el 3.200 AP y el 2.500 AP (Formativo Temprano), aunque podrían llegar a perdurar hasta el 2.000 AP (Salomón Hocsman, comunicación personal 2008).

De esta manera, en nuestra área de estudio hemos hallado 24 puntas de proyectil aparentemente arcaicas tempranas, 38 arcaicas medias, 19 arcaicas tardías y 18 formativas tempranas (ver Tabla 1).

Las 99 puntas han sido encontradas en superficie, en 39 sitios arqueológicos diferentes, emplazados en diversos tipos de unidades topográficas: en la base de cerros y terrazas localizadas en el fondo de la cuenca, en quebradas que comunican Pozuelos con el sur y el oeste, en lagunas y ciénegas de altura, en abras, y en el fondo de la cuenca, alejadas de unidades topográficas destacadas (ver Figura 7). En 23 de los sitios se ha hallado un solo ejemplar, mientras que en los restantes 16 se registraron dos o más ejemplares (ver Tabla 1). Pan de Azúcar 1-3-20 (en la base de un cerro localizado en el fondo de la cuenca) y Laguna Rodeo 1-2 (junto a una laguna de altura) se destacan del resto por la cantidad de puntas de proyectil que en ellos se han encontrado (17 y 13 respectivamente). Laguna Rodeo 1-2 presenta una característica interesante. Allí, en la costa de una laguna de altura actual, hemos hallado un conjunto de artefactos líticos entre los que se encuentran las ocho puntas lanceoladas pequeñas. Tanto desde un punto de vista morfológico como tecnológico, el 


\begin{tabular}{|c|c|c|c|c|c|c|c|c|}
\hline \multirow[b]{2}{*}{ Sitio } & \multirow[b]{2}{*}{$\begin{array}{l}\text { Locali } \\
\text { zación }\end{array}$} & \multirow[b]{2}{*}{$\begin{array}{c}\text { Total } \\
\text { PP }\end{array}$} & \multicolumn{4}{|c|}{$\begin{array}{l}\text { Puntas de proyectil } \\
\text { apedunculadas }\end{array}$} & \multicolumn{2}{|c|}{$\begin{array}{c}\text { Puntas de } \\
\text { proyectil } \\
\text { pedunculadas }\end{array}$} \\
\hline & & & \begin{tabular}{|c|} 
TBR \\
\\
$(10.500$ \\
-9.000 \\
AP) \\
\end{tabular} & $\begin{array}{c}\text { LG } \\
(8.000- \\
7.000 \\
\text { AP) }\end{array}$ & \begin{tabular}{|l} 
LE \\
$(8.000-$ \\
7.000 \\
AP)
\end{tabular} & $\begin{array}{c}\text { LP } \\
\\
(4.000- \\
2.900 \\
\text { AP) }\end{array}$ & $\begin{array}{c}\text { PLTC } \\
(3.200- \\
2.500 \\
\text { AP) }\end{array}$ & $\begin{array}{c}\text { PLTR } \\
(3.200- \\
2.500 \\
\text { AP) }\end{array}$ \\
\hline PA 1-3-20 & CFC & 17 & 4 & $8^{\mathrm{a}}$ & & & 5 & \\
\hline LR 1-2 & LA & 13 & $2^{b}$ & 2 & 1 & 8 & & \\
\hline CCO 1-2 & CFC & 6 & 2 & 2 & & $2^{c}$ & & \\
\hline $\mathrm{CH} 17$ & $\mathrm{FC}$ & 6 & & 1 & & 2 & & 3 \\
\hline RH 49 & TFC & 5 & & 4 & & $1^{\mathrm{d}}$ & & \\
\hline PC 1 & $\mathrm{O}$ & 4 & 3 & & & 1 & & \\
\hline PB 4 & CFC & 3 & 3 & & & & & \\
\hline CIR 1-15 & $\mathrm{CA}$ & 3 & 1 & 1 & & & 1 & \\
\hline AC 4-11 & FC & 3 & & $3^{e}$ & & & & \\
\hline SA 10 & $\mathrm{O}$ & 3 & & 2 & 1 & & & \\
\hline $\begin{array}{l}\text { MPA 303- } \\
\text { C5-C6 }\end{array}$ & CFC & 3 & & 1 & & & $1^{\mathrm{f}}$ & 1 \\
\hline RH 13 & TFC & 2 & 2 & & & & & \\
\hline MPA 604 & CFC & 2 & 1 & & 1 & & & \\
\hline $\mathrm{CCH} 1$ & CFC & 2 & & & & 1 & 1 & \\
\hline TA 1 & $\mathrm{O}$ & 2 & & & & & 1 & 1 \\
\hline CB 9 & CFC & 2 & 1 & 1 & & & & \\
\hline CLC 3 & CFC & 1 & 1 & & & & & \\
\hline CR 10 & CFC & 1 & 1 & & & & & \\
\hline SI C4 & $\mathrm{O}$ & 1 & 1 & & & & & \\
\hline GU P3 & PA & 1 & 1 & & & & & \\
\hline GU 5 & PA & 1 & 1 & & & & & \\
\hline CLG 3 & CFC & 1 & & 1 & & & & \\
\hline $\begin{array}{l}\text { Abra } \\
\text { Colorada }\end{array}$ & $\mathrm{Q}$ & 1 & & 1 & & & & \\
\hline CC 17 & CFC & 1 & & 1 & & & & \\
\hline CLG 9 & CFC & 1 & & 1 & & & & \\
\hline $\mathrm{CH} 8$ & $\mathrm{O}$ & 1 & & 1 & & & & \\
\hline $\begin{array}{l}\text { Laguna } \\
\text { Seca Abra }\end{array}$ & LA & 1 & & 1 & & & & \\
\hline RL 11 & $\mathrm{O}$ & 1 & & 1 & & & & \\
\hline TA 10 & $\mathrm{O}$ & 1 & & 1 & & & & \\
\hline $\mathrm{CH} 7$ & $\mathrm{O}$ & 1 & & & $1^{a}$ & & & \\
\hline CB 5 & CFC & 1 & & & & & $1 \mathrm{~g}$ & \\
\hline CR 50 & CFC & 1 & & & & 1 & & \\
\hline RH 10 & TFC & 1 & & & & & $1 \mathrm{~g}$ & \\
\hline RH 51 & TFC & 1 & & & & & 1 & \\
\hline RH 55 & TFC & 1 & & & $1^{b}$ & & & \\
\hline SI C6 & $\mathrm{O}$ & 1 & & & & $1^{f}$ & & \\
\hline MPA 3 & CFC & 1 & & & & $1 \mathrm{~g}$ & & \\
\hline RH 25 & TFC & 1 & & & & & $1^{\mathrm{f}}$ & \\
\hline GU 15 & $\mathrm{PA}$ & 1 & & & & 1 & & \\
\hline Totales & - & 99 & 24 & 33 & 5 & 19 & 13 & 5 \\
\hline
\end{tabular}

Tabla 1. Puntas de proyectil anteriores al 1.000 AP halladas en nuestra área de estudio. Las puntas fueron confeccionadas con basalto, con excepción de las indicadas. Referencias: a: una punta de cuarcita verde; b: una punta de cuarcita gris; c: una punta de sílice gris; d: una punta de calcita silicificada blanca; e: una punta de cuarcita roja; f: tratamiento unifacial; g: una punta de obsidiana. 8a se lee: "ocho puntas de proyectil, siete confeccionadas con basalto y una con cuarcita verde". FC: fondo de la cuenca; CFC: cerro en el fondo de la cuenca; TFC: terraza en el fondo de la cuenca; Q: quebrada; LA: laguna en franja de altura; CA: ciénega en franja de altura; PA: peña en franja de altura, PP: punta proyectil, TBR: triangulares de base recta, LG: lanceoladas grandes (largo $>5 \mathrm{~cm}$ ); LE: lanceoladas con espolones, LP: lanceoladas pequeñas (largo $<5 \mathrm{~cm}$ ), PLTC: pedunculadas de limbo triangular de lados convexos, PLTR: pedunculadas de limbo triangular recto y bordes dentados. 


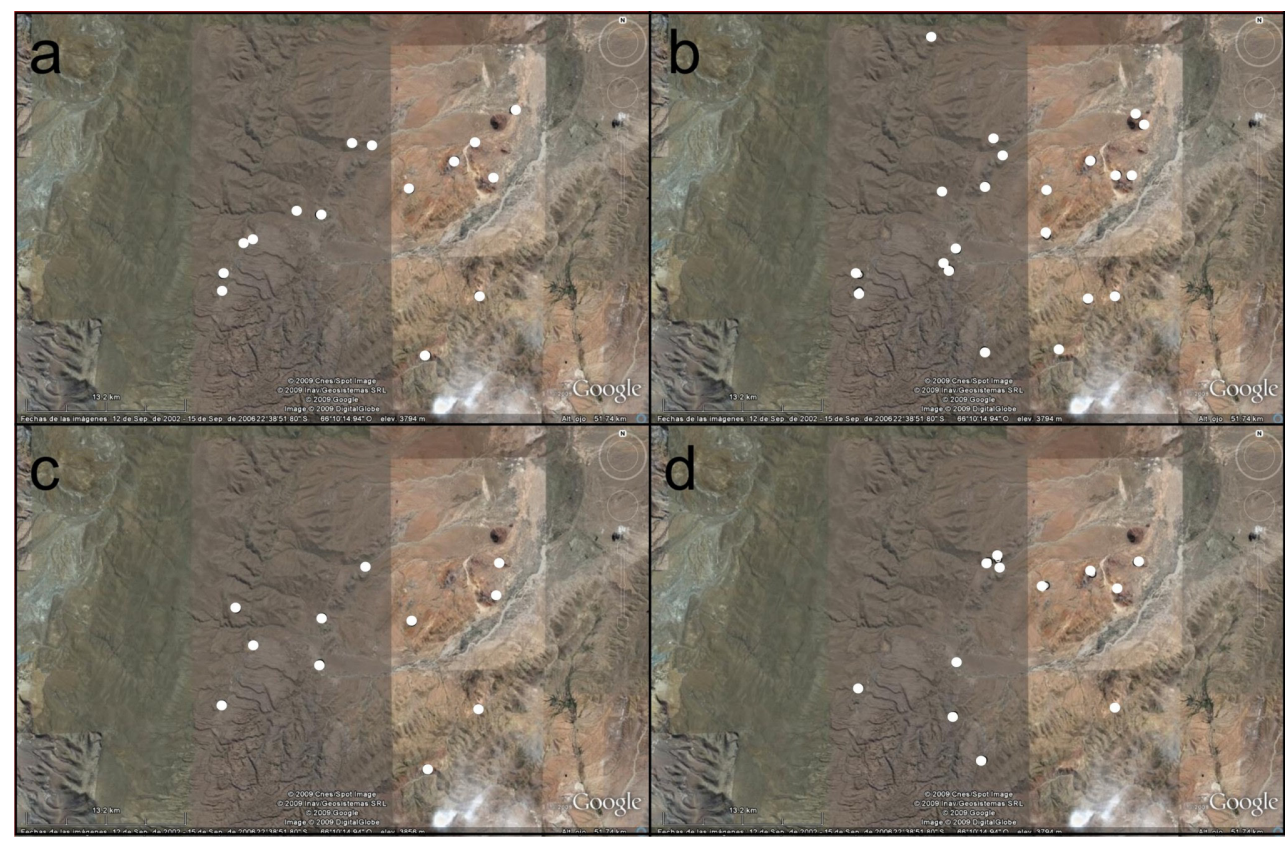

Figura 7. Localización de sitios arqueológicos. Referencias: a: 10.500-9.000 AP; b: 8.000-7.000 AP; c: 4.000-2.900 AP; d: 3.200-2.500 AP. Se han incluido tanto los sitios en los que se han hallado puntas de proyectil como los que presentan representaciones rupestres.

conjunto artefactual recuperado es muy semejante a los hallados en Inca Cueva 7, de época arcaica tardía. Es más, las materias primas empleadas en la confección de los artefactos son muy similares a las utilizadas para la manufactura de las piezas de Inca Cueva 7 (Carlos Aschero comunicación personal 2008).

Hasta el momento hemos registrado 29 sitios en los que se han hallado sólo puntas arcaicas, cinco en los que se han hallado sólo puntas formativas tempranas, y cinco en los que se han hallado puntas de ambas épocas. El hecho de que haya 34 sitios con puntas arcaicas y 10 con puntas formativas tempranas demuestra que las puntas más antiguas presentan una distribución espacial más amplia que las formativas. Tal como en el caso de las representaciones rupestres, tampoco aquí observamos alguna vinculación entre las puntas arcaicas con algún tipo de ambiente o topografía en particular (ver Tablas 2 y 3). Lo que sí está claro es que las puntas lanceoladas grandes, datables entre el $8.000 \mathrm{AP}$ y el 7.000 AP, son las que presentan una dispersión más amplia, encontrándoselas incluso en lagunas de altura localizadas en el extremo noroeste de nuestra área de estudio, donde constituyen las únicas evidencias arqueológicas anteriores a época prehispánica tardía (posterior al 1.200 d.C.). Los sitios con puntas de proyectil de época formativa temprana, en cambio, sí presentan cierta vinculación con determinados ambientes particulares. Ocho de los diez sitios registrados fueron hallados en la base de cerros y terrazas localizados en el fondo de la cuenca. Las puntas de esta época son muy escasas en quebradas (sólo dos ejemplares) y ciénegas de altura (un ejemplar), e inexistentes en lagunas de altura y abras.

Hemos detectado dos casos en los que coinciden el hallazgo de arte rupestre y puntas de proyectil de aproximadamente un mismo momento cronológico. Ellos son: 


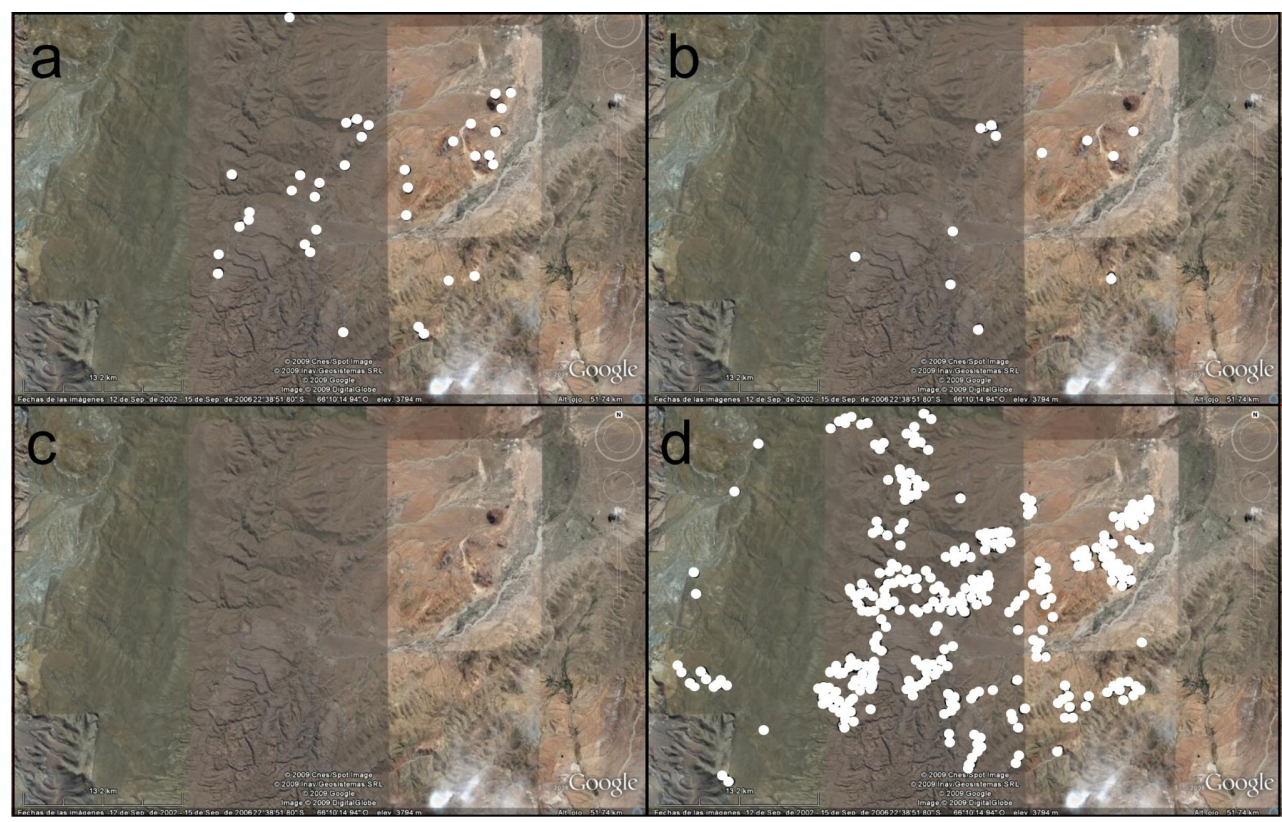

Figura 8. Sitios arqueológicos prehispánicos y coloniales. Referencias: a: arcaicos; b: formativos tempranos; c: del primer milenio de nuestra era; d: posteriores al 1.200 d.C.

\begin{tabular}{|l|c|c|c|c|c|c|c|c|c|c|c|c|}
\hline \multirow{2}{*}{ Localización } & \multicolumn{2}{|c|}{$\mathbf{1 0 . 5 0 0 - 9 . 0 0 0}$ a.p. } & \multicolumn{2}{|c|}{$\mathbf{8 . 0 0 0 - 7 . 0 0 0}$ a.p. } & \multicolumn{2}{|c|}{$\mathbf{4 . 0 0 0 - 2 . 9 0 0}$ a.p. } & \multicolumn{2}{|c|}{$3.200-2.500$ a.p. } \\
\cline { 2 - 13 } & $\begin{array}{c}\mathbf{N}^{\mathbf{0}} \\
\text { sitios }\end{array}$ & $\begin{array}{c}\mathbf{N}^{\mathbf{0}} \\
\text { puntas }\end{array}$ & $\mathbf{A R}$ & $\begin{array}{c}\mathbf{N}^{\mathbf{0}} \\
\text { sitios }\end{array}$ & $\begin{array}{c}\mathbf{N}^{\mathbf{0}} \\
\text { puntas }\end{array}$ & $\mathbf{A R}$ & $\begin{array}{c}\mathbf{N}^{\mathbf{0}} \\
\text { sitios }\end{array}$ & $\begin{array}{c}\mathbf{N}^{\mathbf{0}} \\
\text { puntas }\end{array}$ & $\mathbf{A R}$ & $\begin{array}{c}\mathbf{N}^{\mathbf{0}} \\
\text { sitios }\end{array}$ & $\begin{array}{c}\mathbf{N}^{\mathbf{0}} \\
\text { puntas }\end{array}$ & $\mathbf{A R}$ \\
\hline $\begin{array}{l}\text { Fondo de } \\
\text { cuenca }\end{array}$ & - & - & - & 2 & 4 & - & 1 & 2 & - & 2 & 3 & 1 \\
\hline $\begin{array}{l}\text { Terrazas en } \\
\text { fondo de } \\
\text { cuenca }\end{array}$ & 2 & 2 & 1 & 2 & 5 & - & 1 & 1 & - & 3 & 3 & - \\
\hline $\begin{array}{l}\text { Cerros en fondo } \\
\text { de cuenca }\end{array}$ & 7 & 13 & - & 8 & 16 & - & 5 & 5 & 1 & 4 & 9 & - \\
\hline \begin{tabular}{l} 
Quebradas \\
\hline
\end{tabular} & 2 & 4 & - & 6 & 8 & - & 2 & 2 & 1 & 2 & 2 & 1 \\
\hline $\begin{array}{l}\text { Lagunas en } \\
\text { frania de altura }\end{array}$ & 1 & 2 & - & 2 & 4 & - & 1 & 8 & - & - & - & - \\
\hline $\begin{array}{l}\text { Ciénegas en } \\
\text { frania de altura }\end{array}$ & 1 & 1 & - & 1 & 1 & - & - & - & - & 1 & 1 & 1 \\
\hline $\begin{array}{l}\text { Peñas en franja } \\
\text { de altura }\end{array}$ & 2 & 2 & - & - & - & - & 1 & 1 & - & - & - & - \\
\hline Totales & $\mathbf{1 5}$ & $\mathbf{2 4}$ & $\mathbf{1}$ & $\mathbf{2 1}$ & $\mathbf{3 8}$ & - & $\mathbf{1 1}$ & $\mathbf{1 9}$ & $\mathbf{2}$ & $\mathbf{1 2}$ & $\mathbf{1 8}$ & $\mathbf{3}$ \\
\hline
\end{tabular}

Tabla 2. Localización de los sitios arqueológicos en los que fueron halladas la puntas de proyectil y el arte rupestre. Los sitios con arte rupestre han sido contabilizados también en la columna "No de sitios". AR: Arte rupestre 


\begin{tabular}{|c|c|c|c|c|c|c|c|c|c|c|c|c|}
\hline \multirow[b]{2}{*}{ Localización } & \multicolumn{3}{|c|}{$10.500-9.000$ a.p. } & \multicolumn{3}{|c|}{$8.000-7.000$ a.p. } & \multicolumn{3}{|c|}{ 4.000-2.900 a.p. } & \multicolumn{3}{|c|}{ 3.200-2.500 a.p. } \\
\hline & $\begin{array}{c}N^{0} \\
\text { sitios } \\
\end{array}$ & $\begin{array}{c}\mathbf{N}^{\mathbf{o}} \\
\text { puntas }\end{array}$ & AR & $\mathrm{N}^{\mathrm{o}}$ sitios & \begin{tabular}{|c|}
$\mathbf{N}^{\mathbf{0}}$ \\
puntas
\end{tabular} & AR & $\begin{array}{c}\mathbf{N}^{0} \\
\text { sitios }\end{array}$ & \begin{tabular}{|l|}
$N^{\circ}$ de \\
puntas
\end{tabular} & AR & $\begin{array}{c}\mathbf{N}^{0} \\
\text { sitios }\end{array}$ & \begin{tabular}{|c|}
$\mathbf{N}^{\mathbf{0}}$ \\
puntas
\end{tabular} & AR \\
\hline $\begin{array}{l}\text { Fondo de } \\
\text { cuenca }\end{array}$ & 9 & 15 & 1 & 12 & 25 & - & 7 & 8 & 1 & 9 & 15 & 1 \\
\hline Quebradas & 2 & 4 & - & 6 & 8 & - & 2 & 2 & 1 & 2 & 2 & 1 \\
\hline $\begin{array}{l}\text { Franja de } \\
\text { altura }\end{array}$ & 4 & 5 & - & 3 & 5 & - & 2 & 9 & - & 1 & 1 & 1 \\
\hline Totales & 15 & 24 & 1 & 21 & 38 & - & 11 & 19 & 2 & 12 & 18 & 3 \\
\hline
\end{tabular}

Tabla 3. Localización de los sitios arqueológicos en los que fueron halladas la puntas de proyectil y el arte rupestre. Los sitios con arte rupestre han sido contabilizados también en la columna " $\mathrm{N}^{\mathrm{O}}$ de sitios

-Peña Colorada 1, localizado en la cima de una peña, junto a un abra que comunica nuestra área con el sur, en el que se ha encontrado arte asignable al Arcaico Tardío y una punta de la misma época (aunque allí predominan las arcaicas tempranas); y

-Ciénega Rodeo 1-15, localizado en una ciénega de altura, en el sector occidental de nuestra área, en el que se han encontrado una punta de proyectil y representaciones rupestres que datarían de época formativa temprana. En este sitio, además, abundan el arte y los materiales tardíos en superficie (es el único caso en el que se observa esta recurrencia).

En lo que respecta a las materias primas con que fueron confeccionadas las puntas de proyectil, podemos señalar que la enorme mayoría ha sido tallada en basalto (88 de 99 ejemplares, ver Tabla 1). Para la manufactura de las restantes se ha utilizado cuarcita (en tres casos de puntas arcaicas, dos lanceoladas grandes y una lanceolada con espolones), alguna variedad de sílice (en dos casos de puntas arcaicas tardías, ambas lanceoladas pequeñas) y obsidiana (en dos casos de puntas formativas, ambas pedunculadas de limbo triangular de lados convexos). Para época prehispánica tardía (posterior al 1.200 d.C.), a diferencia de lo que ocurre con los casos que hemos señalado, no hemos hallado puntas confeccionadas con basalto, sino que comienzan a utilizarse como materia prima cuarzo y diversas variedades de sílice (muchas de ellas locales), y aumenta notablemente el uso de obsidiana.

En referencia a las posibles fuentes de materias primas líticas para la manufactura de las puntas de proyectil presentadas, las cuarcitas, supuestamente ajenas a la geología de la zona, ya que no aparecen mapeadas ni nombradas en los informes geológicos (p.ej. Coira 1979), podrían llegar a configurarse como material local debido a que se presentan en forma de rodados en los lechos de los ríos de la zona. Es más, existe la posibilidad de que se encuentren en nuestra área de estudio pequeños afloramientos que por sus reducidas dimensiones no hayan sido mapeados en estudios regionales (Rivet 2007). Consideramos, entonces, la posibilidad de que la zona de aprovisionamiento de este recurso lítico se encuentre en los alrededores de las formaciones dómicas que se hallan en el sector de fondo de cuenca del área. Lo mismo sucede en relación a los sílices, que también forman parte de la geología local. Por el contrario, no fue registrado en la zona de estudio ninguna posible fuente de basalto ni de obsidiana. Estos recursos proceden de lugares más alejados, probablemente localizados hacia el oeste de nuestra área. Específicamente en el caso de las puntas formativas de obsidiana, por sus características observables macroscópicamente ambas habrían sido confeccionadas con materia prima procedente de la fuente de Vilama (Alfredo Calisaya comunicación personal 2009). 


\section{Consideraciones finales}

En base a las evidencias encontradas hasta el momento, notamos una ocupación arcaica más visible y extendida que la formativa en el sector de estudio, sobre todo si tenemos en cuenta la cantidad de sitios arqueológicos en los que fueron halladas evidencias arcaicas (36) versus la cantidad de sitios arqueológicos en los que fueron halladas evidencias de época formativa (12), la diversidad de ambientes en las que se encuentran los primeros, versus la uniformidad de paisajes en las que se encuentran los segundos, y la cantidad de puntas que podemos asignar a época arcaica (81) versus las que podemos asignar a época formativa (18). Por otro lado, el registro para este último período indicaría fundamentalmente una ocupación formativa temprana, con aún menor visibilidad para el primer milenio de nuestra era. Cuando avancemos en el estudio de las puntas que hemos asignado tentativamente a época prehispánica tardía (posterior al 1.000 d.C.), podría darse el caso de que algunas de ellas fueran en realidad un poco más antiguas. Sin embargo, por lo que hemos analizado hasta ahora, no serían muchas.

La disminución paulatina de la cantidad de evidencias arqueológicas desde el Arcaico Temprano hasta el Formativo Temprano, la ausencia de materiales asignables al primer milenio de nuestra era, y el incremento exponencial de evidencias de todo tipo para después del 1.200 d.C. (ver Figura 8), es ahora uno de nuestros temas a abordar en el futuro. En el estado actual de nuestras investigaciones no podemos explicar esta situación. En términos generales se podría pensar que una disminución en la manufactura de puntas de proyectil podría asociarse a un menor énfasis en las prácticas de caza, con mayor predominio relativo de actividades productivas como la agricultura y el pastoreo. Sin embargo, no hay evidencias que sustenten la hipótesis de un crecimiento de tales actividades en época formativa. Por el contrario, sospechamos un relativo despoblamiento del sur de Pozuelos durante gran parte del primer milenio de nuestra era (Angiorama 2010). La falta de arquitectura que podamos asignar a aquella época, y la casi total ausencia de cerámica del primer milenio de nuestra era en las muestras que hemos recolectado, son elementos que enfatizan aún más este panorama. Estudios geomorfológicos, paleoambientales y tafonómicos como los mencionados antes, sumados a análisis más profundos de los elementos presentados en este trabajo, permitirán configurar un marco más adecuado para evaluar la significación de los hallazgos presentados en esta oportunidad.

Agradecimientos: La investigación realizada ha sido posible gracias a subsidios otorgados por CONICET y FONCyT. Agradecemos a Carlos Aschero, Axel Nielsen, Jorge Martínez y Salomón Hocsman por la ayuda que nos han brindado durante la preparación de este trabajo, y a cada uno de los que han participado en los trabajos de campo realizados en Pozuelos: Jorge Martínez, Constanza Taboada, Paulo Barrales, Luciana Chavez, Alexis Coronel, Flavia Germano, Iván Fernández, Lorena Pérez, Carolina Rivet, Bruno Salvatore, Gustavo Spadoni, Antonino Taboada y José Luis Tolaba. Agradecemos también a Humberto Mamaní por el apoyo otorgado a nuestro trabajo, y a los habitantes de Pozuelos, que hacen que cada momento compartido sea inolvidable. Ninguno de ellos, sin embargo, es responsable de lo expresado en este artículo.

\section{Nota}

1. Jornadas de Arqueología del Área Puneña de Los Andes Centro-Sur. Tendencias, Variabilidad y Dinámicas de Cambio (ca.11000-1000 AP). Instituto de Arqueología y Mu- 
seo, Universidad Nacional de Tucumán, e Instituto Superior de Investigaciones Sociales, CONICET. Horco Molle (Tucumán), agosto de 2008.

\section{Referencias Citadas}

Agüero, C., P. Ayala, M. Uribe, C. Carrasco y B. Cases

2006 El Período Formativo desde Quillagua, Lao Inferior (norte de Chile). En Esferas de Interacción Prehistóricas y Fronteras Nacionales Modernas: Los Andes Sur Centrales, editado por H. Lechtman, pp. 73-126. IEP-IAR, Lima-Nueva York.

Alfaro de Lanzone, L. y J. Suetta

1970 Nuevos aportes para el estudio del asentamiento humano en la puna de Jujuy. Revisión del Pucará de Rinconada. Antiquitas 10:1-10.

Ambrosetti, J.

1902 Antigüedades Calchaquíes. Datos Arqueológicos sobre la Provincia de Jujuy. Anales de la Sociedad Científica Argentina, tomos LII, LIII y LIV. Buenos Aires.

Angiorama, C. I.

2001 De metales, minerales y yacimientos. Contribución al estudio de la metalurgia prehispánica en el extremo noroccidental de Argentina. Estudios Atacameños 21:63-87.

2003 Producción y Circulación de Objetos de Metal en la Quebrada de Humahuaca en Momentos Prehispánicos Tardíos (900-1535 d.C.). Tesis Doctoral inédita. Facultad de Ciencias Naturales e Instituto M. Lillo, Universidad Nacional de Tucumán, San Miguel de Tucumán.

2006 ¿Mineros quebradeños o altiplánicos? La circulación de metales y minerales en el extremo noroccidental de Argentina (1280 - 1535 A.D.). Intersecciones en Antropología 7:147-161. 2009 La presencia del IAM en la Puna jujeña: el proyecto arqueológico Sur de Pozuelos. En Rastros en el Camino. Trayectos e Identidades de una Institución, editado por C. Aschero, P. Arenas y C. Taboada. Pp. 321-328. Editorial de la Universidad Nacional de Tucumán, San Miguel de Tucumán.

2010. La ocupación del espacio en el sur de Pozuelos (Jujuy, Argentina) durante tiempos prehispánicos y coloniales. Estudios Sociales del NOA 11: 125-142.

Aschero, C. A.

1979 Aportes al estudio del arte rupestre de Inca Cueva 1 (Dpto. Humahuaca, Jujuy). Actas de las Jornadas de Arqueología del Noroeste Argentino. Universidad del Salvador, Buenos Aires.

2007 Iconos, huancas y complejidad en la Puna sur Argentina. En Producción y circulación prehispánicas de bienes en el sur andino, compilado por A. E. Nielsen, M. C. Rivolta, V. Seldes, M.M. Vázquez y P. Mercolli, pp. 135-165. Editorial Brujas, Córdoba.

Aschero, C.A. y M.M. Podestá.

$1986 \mathrm{El}$ arte rupestre en asentamientos precerámicos en la puna Argentina. Runa 16:29-57.

Boman, E.

1992 [1908] Antigüedades de la Región Andina de la República Argentina y del Desierto de Atacama. UNJu, San Salvador de Jujuy. 
Coira, B.

1979 Descripción geológica de la Hoja 3c, Abra Pampa. Provincia de Jujuy. Escala 1: 200000. Boletín n ${ }^{\circ} 170$. Servicio Geológico Nacional. Buenos Aires.

Fernández, J.

1996 Munitayoc, nuevo sitio con cerámica temprana (1000 a.C.) en la Puna jujeña. Actas y Memorias del XI Congreso Nacional de Arqueología Argentina (11 ${ }^{\circ}$ parte) Revista del Museo de Historia Natural de San Rafael Tomo XXIII (1/4):51-62.

Krapovickas, P.

1975 Algunos tipos cerámicos de Yavi Chico. Actas y Trabajos del Primer Congreso Nacional de Arqueología Argentina (Rosario, 1970), pp. 293-300. Buenos Aires.

Hocsman, S.

2006 Producción Lítica, Variabilidad y cambio en Antofagasta de la Sierra -ca.5500-1500 AP. Tesis para optar al grado de Dr. en Ciencias Naturales, Orientación Arqueología. Facultad de Ciencias Naturales y Museo, Universidad Nacional de La Plata, La Plata.

2010 Cambios en las puntas de proyectil durante la transición de cazadores-recolectores a sociedades agro-pastoriles en Antofagasta de la Sierra (Puna argentina). Revista Arqueología 16:59-86. Buenos Aires.

Lecoq, P.

2001 El Período Formativo en Potosí y el sur de Bolivia: un estado de la cuestión. Textos Antropológicos 13(1-2):231-263.

Lupo, L., M. R. Morales, H. D. Yacobaccio, A. Maldonado y M. Grosjean

2007 Cambios ambientales en la puna jujeña durante los últimos 1200 años: explorando su impacto en la economía pastoril. Actas del XVI Congreso Nacional de Arqueología Argentina, Tomo III, pp. 151-156. Universidad Nacional de Jujuy. San Salvador de Jujuy.

Martínez, J.

2003 Ocupaciones Humanas Tempranas y Tecnología de caza en la Microrregión de Antofagasta de la Sierra (10000 a 7000 AP). Tesis para optar al grado de Dr. en Arqueología. Facultad de Ciencias Naturales e Instituto M. Lillo, Universidad Nacional de Tucumán, San Miguel de Tucumán.

Nielsen, A. E.

1997 Tiempo y Cultura Material en la Quebrada de Humahuaca. 700-1650 d.C. Instituto Interdisciplinario Tilcara. Facultad de Filosofía y Letras, Universidad Nacional de Buenos Aires.

Olivera, D., P. Tchilinguirian y L. Grana

2004 Paleoambiente y arqueología en la Puna Meridional Argentina: archivos ambientales, escalas de análisis y registro arqueológico. Relaciones de la Sociedad Argentina de Antropología XXIX:229-247.

Ottonello, M.

1973 Instalación, economía y cambio cultural en el sitio Tardío de Agua Caliente de Rachaite. Publicaciones de la Dirección de Antropología e Historia nº 1:23-68. San Salvador de Jujuy. 
Ottonello, M y P. Krapovickas

1973 Ecología y arqueología de cuencas en el sector oriental de la Puna, República Argentina. Publicaciones de la Dirección de Antropología e Historia nº 1:3-21. San Salvador de Jujuy.

Raffino, R. A., R. J. Alvis, D. E. Olivera y J. R. Palma

1986 La instalación inka en la sección andina meridional de Bolivia y extremo boreal de Argentina. En El imperio Inka: actualización y perspectiva. Comechingonia Número especial, editado por R. A. Raffino, pp. 63-129.

Rivet, C.

2007 Identificación de las materias primas líticas del área arqueológica Pan de Azúcar (Rinconada, Jujuy). Informe inédito: Cátedra Práctica de Campo IV de la Carrera de Arqueología, Universidad Nacional de Tucumán. San Miguel de Tucumán. Manuscrito en posesión de la autora.

Ruiz, M.

1996 Algunas reflexiones sobre las agrupaciones G-I-K del Pucará de Rinconada, Puna de Jujuy, República Argentina. XXX Aniversario Museo Arqueológico Dr. Eduardo Casanova, pp. 137-144. Instituto Interdisciplinario Tilcara, Facultad de Filosofía y Letras, Universidad de Buenos Aires, Tilcara.

Ruiz, M. y M.E. Albeck

1992 El fenómeno pukara visto desde la puna jujeña. Cuadernos 9:233-255.

Sinclaire, C.

2004 Prehistoria del Período Formativo en la cuenca alta del Río Salado (Región del Loa Superior). Chungara 36(2):619-639.

Suetta, J. y L. Alfaro de Lanzone

1979 Excavaciones Arqueológicas en el pucará de Rinconada, Pcia. de Jujuy. Actas de las Jornadas de Arqueología del Noroeste Argentino. Universidad del Salvador, Buenos Aires.

Tecchi, R.

1991 Los ecosistemas Puna y Suni en la cuenca de la Laguna Pozuelos. En La Reserva de la Biosfera Laguna Pozuelos: un Ecosistema Pastoril en los Andes Jujeños, compilado por J. García Fernández y R Tecchi, pp. 9-22. Universidad Nacional de Jujuy. San Salvador de Jujuy. 
